\title{
ENTROPIC APPROXIMATION IN KINETIC THEORY
}

\author{
JACQUES SCHNEIDER ${ }^{1}$
}

\begin{abstract}
Approximation theory in the context of probability density function turns out to go beyond the classical idea of orthogonal projection. Special tools have to be designed so as to respect the nonnegativity of the approximate function. We develop here and justify from the theoretical point of view an approximation procedure introduced by Levermore [Levermore, J. Stat. Phys. 83 (1996) 1021-1065] and based on an entropy minimization principle under moment constraints. We prove in particular a global existence theorem for such an approximation and derive as a by-product a necessary and sufficient condition for the so-called problem of moment realizability. Applications of the above result are given in kinetic theory: first in the context of Levermore's approach and second to design generalized BGK models for Maxwellian molecules.
\end{abstract}

Mathematics Subject Classification. 54C70, 46N10, 82B40, 37L65.

Received: February 4, 2003.

\section{INTRODUCTION}

The mathematical entropy $H(f)=\int f \ln f-f$ plays an important role in kinetic theory. Boltzmann showed that it expresses the irreversibility of the evolution of a system of infinitely many particles subjected to their own interaction. Secondly it measures the distance of such a system to the fluid limit. Finally among all other meanings and uses of the entropy it can be used to reconstruct from macroscopic values the microscopic description of the density function at local equilibrium (Maxwellian distribution). We are going to study in this article the generalization of this last principle, that is, the possibility to reconstruct a density function from a given set of moments (in the velocity space) of that density function. Such a generalization supposes, first, that the set of moments, which we denote by $\rho$, is that of a nonnegative density function $f$ unless there is no possible kinetic interpretation. That is, $\rho=\int \mathbf{m}(\mathbf{v}) f(\mathbf{v}) \mathrm{d} \mathbf{v}$ with $f \geq 0$ and where $\mathbf{m}(\mathbf{v})$ is a vector whose components can be for example polynomials of $\mathbf{v}$. This will be an a priori assumption in our work. Then, we follow the idea of Levermore that consists in reconstructing a function $G$ from the moments $\rho$ through an entropy minimization principle [11]. Notice that such a principle is also used within the context of hyperbolic systems, that is, at a macroscopic level to define entropic projections suited to numerical approximation $[3,4]$. However in that context the space on which the projection is defined (piecewise linear functions) is set a priori while here it is not and is rather defined a posteriori.

We start our work (Sect. 3) by proving the following result: given a nonnegative function $f$ with bounded moments of order $N$ and a polynomial space $\mathbf{P}=\mathbf{P}_{N-1} \oplus \mathbb{R}$. $|\mathbf{v}|^{N}$, there exists always a function $G$ that is the

Keywords and phrases. Kinetic entropy, convex analysis, nonlinear approximation, moments systems, Maxwellian molecules.

1 Laboratoire Modélisation Numérique et Couplages, Université de Toulon et du Var, 83162 La Valette Cedex, France.

e-mail: jacques.schneider@univ-tln.fr 
solution to the minimization problem

$$
\min \left\{H(g): \int m(\mathbf{v})(g-f) \mathrm{d} \mathbf{v}=0 \forall m \in \mathbf{P}_{N-1}, \quad \int g|\mathbf{v}|^{N} \leq \int f|\mathbf{v}|^{N}\right\}
$$

Moreover this solution is unique and can be written as $G(\mathbf{v})=\exp \left(m(\mathbf{v})+\alpha|\mathbf{v}|^{N}\right)$ where $m \in \mathbf{P}_{N-1}$ and $\alpha \leq 0$ are uniquely determined by the above moments constraints. We call the application $f \rightarrow \rho \rightarrow G$ an entropic approximation of $f$ on $\exp (\mathbf{m})$. This result is more or less equivalent to the work of Csiszár [5] concerning I-projection where a probabilistic approach is used and later on to Léonard [10] where duality in Orlics spaces is the main tool. But in our proof pure functional (convex) analysis is preferred (see Rem. 2). This result is also more or less equivalent to that of Junk $[8,9]$ and in some sense more directly obtained. Besides the originality here is that the constraint of highest degree is relaxed so as to close the convex set of constraints and ensure a global existence result for the minimization problem.

The entropic approximation finds a first application (Sect. 4) within the approach of Levermore concerning moment closure in kinetic theory where it is used to define approximation of kinetic equations. The only difference is that in Levermore's approach all constraints must be qualified. We derive from our general result a necessary and sufficient condition for qualification of all constraints, henceforth giving the domain of definition of Levermore's approach and recovering the results of Junk.

A second important application of the H-projection is a generalization of the so-called BGK relaxation model for the Boltzmann equation (Sect. 5). It allows us to construct relaxation terms of the form $\nu(G-f)$ that fit exactly to the Boltzmann collision operator $Q(f, f)$ for Maxwellian molecules in the following weak sense:

$$
\nu \int(G-f) m(\mathbf{v}) \mathrm{d} \mathbf{v}=\int Q(f, f) m(\mathbf{v}) \mathrm{d} \mathbf{v} \quad \forall m \in \mathbf{P},
$$

with $\nu=\int f$. Notice here that $\mathbf{P}$ is an arbitrarily big polynomial space and that $G$ is the entropy minimizer whose moments in $\mathbf{P}$ are those of the positive part $\left(\int f\right)^{-1} Q^{+}(f, f)$ of the Boltzmann collision operator. Consequently $\nu(G-f)$ can be considered as an approximation of $Q(f, f)$ whose order depends on $\mathbf{m}$. Important advantages are:

(1) The costly computation of the 5-fold collision integral at each velocity (see e.g. [14] for numerical issues) is here replaced by the computation of a single minimization problem which is valid for all velocities this minimization process has already shown its efficiency for the classical BGK model (where $G$ is the Maxwellian distribution) within the discrete velocity approach [12].

(2) The fluid limit remains correct provided the relaxation rates of moments of order less than $N$ are correct. Consequently such models inherit the same viscosity, heat flux transfer or Prandtl number as the Boltzmann fluid limit. Moreover the behavior of such moments are good even far from equilibrium due to (1).

After deriving the models we prove in this section that due to the minimization principle and to recent results on entropy dissipation ([15] and references therein) such models also enjoy the entropy dissipation law

$$
\nu \int(G-f) \ln f \mathrm{~d} \mathbf{v} \leq 0
$$

and we then study the Chapman-Enskog asymptotic of these models. 


\section{Notations}

- $\langle g\rangle$ is the Lebesgue measure of all measurable function $g$ :

$$
\langle g\rangle=\int_{\mathbb{R}^{3}} g(\mathbf{v}) \mathrm{d} \mathbf{v}
$$

with possible value $\infty$.

- $L_{N}^{1}\left(\mathbb{R}^{d}\right)$ is the Banach subspace of $L^{1}$ defined by

$$
L_{N}^{1}\left(\mathbb{R}^{d}\right)=\left\{f \in L^{1} \text { such that }\left\langle|f(\mathbf{v})|\left(1+|\mathbf{v}|^{2}\right)^{N / 2}\right\rangle<+\infty\right\}
$$

provided with the norm

$$
\|f\|_{L_{N}^{1}}=\left\|\left(1+|\mathbf{v}|^{2}\right)^{N / 2} f\right\|_{L^{1}} .
$$

Its dual is $L_{-N}^{\infty}$, the space of functions growing not faster than $|\mathbf{v}|^{N}$ at infinity.

- $\mathbf{P}_{N}\left(\mathbb{R}^{d}\right)$ is the space of polynomials of order less than or equal to $N$ in $\mathbb{R}^{d}$. Let

$$
\mathbf{v}^{\mathbf{q}}=v_{1}^{q_{1}} v_{2}^{q_{2}} \ldots v_{d}^{q_{d}}, \mathbf{q}=\left(q_{1}, q_{2}, \ldots, q_{d}\right)^{T} \in \mathbf{N}^{d}
$$

then the degree of the monomial $\mathbf{v}^{\mathbf{q}}$ is $|\mathbf{q}|=q_{1}+q_{2}+\ldots+q_{d}$ and $\mathbf{v}^{\mathbf{q}} \in \mathbf{P}_{N}$ iff $|\mathbf{q}| \leq N$. Denoting by $\# N=\left(\begin{array}{c}N+d \\ N\end{array}\right)$ the dimension of the vector space $\mathbf{P}_{N}$ and with $\mathbf{m}(\mathbf{v})$ the application from $\mathbb{R}^{d} \rightarrow \mathbb{R}^{N}$ whose components are all monomials $\mathbf{v}^{\mathbf{q}}$ of order less than $N$ then every $p \in \mathbf{P}_{N}$ can be written as

$$
p(\mathbf{v})=\boldsymbol{\alpha} \cdot \mathbf{m}(\mathbf{v}) \text { with } \boldsymbol{\alpha} \in \mathbb{R}^{\# N} .
$$

- $\boldsymbol{\mu}$ denotes the linear and continuous application from $L_{N}^{1}\left(\mathbb{R}^{d}\right)$ into $\mathbb{R}^{\# N}$ defined by

$$
\forall f \in L_{N}^{1}\left(\mathbb{R}^{d}\right), \quad \boldsymbol{\mu}(f)=\langle\mathbf{m}(\mathbf{v}) f(\mathbf{v})\rangle .
$$

- Likewise to any polynomial space $\mathbf{m} \subset \mathbf{P}_{N}$ of finite dimension $\# P$ and of degree $N$, we associate the \#P-vector whose components are its generating polynomials (not necessarily monomials) and the corresponding measure $\boldsymbol{\mu}(f)=\langle\mathbf{m}(\mathbf{v}) f(\mathbf{v})\rangle$.

- For fixed $\mathbf{P}$ we denote with $\leq^{*}\left(\right.$ resp. $\left.\geq^{*}\right)$ the order relation:

$$
\begin{array}{r}
\forall f, g \in L_{N}^{1}: \boldsymbol{\mu}(f) \leq^{*} \boldsymbol{\mu}(g)\left(\text { resp. } \geq^{*}\right) \Longleftrightarrow \\
\mu_{\mathbf{q}}(f)=\mu_{\mathbf{q}}(g) \text { if }|\mathbf{q}| \leq N-1,\left\langle f|\mathbf{v}|^{N}\right\rangle \leq\left\langle g|\mathbf{v}|^{N}\right\rangle(\text { resp. } \geq)
\end{array}
$$

\section{Entropic Approximation: the GENERAL RESUlt}

Let $H$ denote the entropy functional acting on $L^{1}$ functions defined as:

$$
H(f)=\left\{\begin{array}{l}
\langle f \ln f-f\rangle \text { if } f \geq 0 \\
+\infty \text { otherwise. }
\end{array}\right.
$$

We are interested in finding a solution to the problem of minimizing the entropy under moment constraints. That is we are looking for solution(s) to

$$
\min \{H(g), \quad \boldsymbol{\mu}(g)=\rho\}
$$


where $\boldsymbol{\mu}$ is the above measure associated to the polynomial space $\mathbf{m}=\mathbf{P}_{N-1} \oplus \mathbb{R}|\mathbf{v}|^{N}$. This problem can be divided into two parts:

(1) Clearly due to the definition of $H$ this problem makes sense if and only if the set of constraints $\{f \geq$ $0, \boldsymbol{\mu}(f)=\rho\}$ is not empty that is if $\rho \in \boldsymbol{\mu}\left(\left(L_{N}^{1}\right)^{+}\right)$where $\left(L_{N}^{1}\right)^{+}$is the cone of $L_{N}^{1}$-nonnegative functions:

$$
\left(L_{N}^{1}\right)^{+}=\left\{g \in L_{N}^{1}\left(\mathbb{R}^{d}\right), g \geq 0 \text { a.e. }\right\}
$$

Such a problem is known as the Hamburger moment problems and there exists already an extensive literature concerning it (see [8] and references therein). Remark however that if we define $\rho$ as the moments $\boldsymbol{\mu}(f)$ of the solution to some kinetic equation then this problem does not arise and the only thing to check is that this solution has bounded moments.

(2) Suppose that $\rho \in \boldsymbol{\mu}\left(\left(L_{N}^{1}\right)^{+}\right)$then one wants to find among all functions $f \in\left(L_{N}^{1}\right)^{+}$such that $\boldsymbol{\mu}(f)=\rho$ the one(s) with minimal entropy.

Our main result consists in proving that the minimization problem for $\rho \in \boldsymbol{\mu}\left(\left(L_{N}^{1}\right)^{+}\right)$always possesses a solution whenever the constraint of highest degree is relaxed. More precisely we prove the following theorem:

Theorem 1. Let $N \in \mathbf{N}$ and $f$ be a non-negative function in $L_{N}^{1}\left(\mathbb{R}^{d}\right)$ such that $H(f)<+\infty$. Set

$$
C_{f}=\left\{g \geq 0, \boldsymbol{\mu}(g) \leq^{*} \boldsymbol{\mu}(f)\right\}
$$

then the minimization problem

$$
\min _{g \in C_{f}} H(g)
$$

possesses a unique solution in $C_{f}$ which takes the general form

$$
G(\mathbf{v})=\exp (\boldsymbol{\alpha} \cdot \mathbf{m}(\mathbf{v}))
$$

where the Lagrange multipliers $\boldsymbol{\alpha}$ are uniquely determined by the moment constraints $\boldsymbol{\mu}(G) \leq^{*} \boldsymbol{\mu}(f)$. If the last constraint is not qualified and $N$ is odd then $\boldsymbol{\alpha} \cdot \mathbf{m}(\mathbf{v}) \in \mathbf{P}_{N-1}$ and $G$ is a global minimizer of $H$ in $L_{N-1}^{1}$ under the only constraints $\mu_{\mathbf{q}}(G)=\mu_{\mathbf{q}}(f), \quad|\mathbf{q}| \leq N-1$. If $N$ is even then $\boldsymbol{\alpha} \cdot \mathbf{m}(\mathbf{v}) \in \mathbf{P}_{N-2}$ and $\mu_{\mathbf{q}}(G)=\mu_{\mathbf{q}}(f), \quad|\mathbf{q}| \leq N-2$.

Conversely if there exists in $C_{f}$ a function of the form $\exp (m(\mathbf{v}))$ with $m \in \mathbf{P}$ then such a function is unique and it is the solution to our minimization problem.

Definition 1. The application $f \longrightarrow G$ is called an entropic approximation on $\exp (\mathbf{P})$.

We shall see in the next section that the case of the minimization problem with qualified constraints can be seen as a consequence of that result. So let us now turn to the proof of Theorem 1. We first recall some technical properties on $H$ that have already been proved in different contexts $[2,6,8]$ and we prove them for the sake of consistency.

\section{Lemma 1.}

(1) Let $f \in L_{N}^{1}\left(\mathbb{R}^{d}\right), N \geq 2$, then $H(f)>-\infty$;

(2) $H$ is convex lower semi-continuous (l.s.c) in $L_{N}^{1}\left(\mathbb{R}^{d}\right), N \geq 2$;

(3) let $B$ be a bounded set in $L_{N}^{1}\left(\mathbb{R}^{d}\right)$ such that $H$ is bounded on $B$, then $B$ is a weakly (relatively) compact in $L^{1}\left(\mathbb{R}^{d}\right)$.

Proof. (i) For every $f \in L_{N}^{1}\left(\mathbb{R}^{d}\right)$, one can control the negative part of $H$ by the $L_{N}^{1}$ norm:

$$
\int_{0 \leq f \leq 1} f \ln \frac{1}{f} \mathrm{~d} \mathbf{v} \leq \int_{\mathbb{R}^{d}} f|\mathbf{v}|^{2}+\int_{0 \leq f \leq e^{-v^{2}}} f \ln \frac{1}{f} \mathrm{~d} \mathbf{v} .
$$


But for $0 \leq x \leq 1$, there exists $C>0$ such that $x \ln 1 / x \leq C \sqrt{x}$. Hence:

$$
H(f) \geq-\|f\|_{L_{N}^{1}}-C \int \mathrm{e}^{-v^{2} / 2}=-\|f\|_{L_{N}^{1}}-C_{1}
$$

where $C_{1}$ is a positive constant independent of $f$.

(ii) Due to the convexity of $H$, it is enough to prove that $H$ is l.s.c for the strong topology. So let $f_{n} \rightarrow f$ in $L_{N}^{1}$. Either $\lim \inf H\left(f_{n}\right)=+\infty$ and consequently $H(f) \leq \liminf H\left(f_{n}\right)$ or $\liminf H\left(f_{n}\right)=\alpha<+\infty$. Then up to successive extractions of sub-sequences, one has:

$$
H\left(f_{n}\right) \rightarrow \alpha, f_{n} \rightarrow f \text { in } L_{N}^{1} \text { and almost everywhere. }
$$

But $H\left(f_{n}\right)<+\infty \Rightarrow f_{n} \geq 0$ and passing to the limit $f \geq 0$. Then due to the continuity of $x \ln x$ on $\mathbb{R}^{+}$:

$$
f_{n} \ln f_{n} \rightarrow f \ln f \text { a.e. }
$$

Now remark that the inequality $x \ln x \geq-\mathrm{e}^{-1}$ implies $x \ln \left(x \mathrm{e}^{v^{2}}\right) \geq-\frac{1}{e} \mathrm{e}^{-v^{2}}$ or

$$
x \ln x+x v^{2}+\frac{1}{e} \mathrm{e}^{-v^{2}} \geq 0, \quad \forall x \geq 0, \forall \mathbf{v} \in \mathbb{R}^{d} .
$$

Setting $h_{n}=f_{n} \ln f_{n}+f_{n} v^{2}+\frac{1}{e} \mathrm{e}^{-v^{2}}$ one has the following properties:

$$
h_{n} \geq 0, h_{n} \rightarrow h=f \ln f+f v^{2}+\frac{1}{e} \mathrm{e}^{-v^{2}} \text { a.e., } \int h_{n} \mathrm{~d} \mathbf{v}<+\infty .
$$

Using Fatou lemma one obtains:

$$
\int h \leq \liminf \int h_{n}
$$

and since $f_{n} \rightarrow f$ this gives

$$
H(f) \leq \liminf H\left(f_{n}\right)
$$

That is, $H$ is l.s.c for the strong topology and by convexity for the weak topology $\sigma\left(L_{N}^{1}, L_{-N}^{\infty}\right)$.

(iii) According to (i) $H$ is bounded from below on $B$ and so:

$$
\int_{\mathbb{R}^{d}} g\left(1+|\mathbf{v}|^{N}+|\ln g|\right) \mathrm{d} \mathbf{v}<+\infty, \quad \forall g \in B
$$

from which one deduces as in [6] that $B$ is "equi-integrable" in the sense of Dunford-Pettis lemma and consequently weakly relatively compact in $L^{1}$.

The second lemma sets the problem of minimization of entropy on bounded sets of velocity. We introduce for this particular case the notations:

$$
H_{K}(f)=\int_{\Omega}(f \ln f-f) \mathrm{d} \mathbf{v}, \quad \boldsymbol{\mu}^{K}(f)=\int_{K} \mathbf{m}(\mathbf{v}) f \mathrm{~d} \mathbf{v}
$$

where $K$ is any set in $\mathbb{R}^{d}$. In that case all constraints are automatically qualified as long as $\rho \in \boldsymbol{\mu}^{K}\left(\left(L^{1}(K)\right)^{+}\right)$.

Lemma 2. Let $K \subset \mathbb{R}^{d}$ be a compact set and let $f$ be a nonnegative function in $L^{1}(K)(f \neq 0)$. Then there exists a unique solution to the minimization problem

$$
\min \left\{H(g): \boldsymbol{\mu}^{K}(g)=\boldsymbol{\mu}^{K}(f)\right\} .
$$


This solution takes the form:

$$
G(\mathbf{v})=\exp (\boldsymbol{\alpha} \cdot \mathbf{m}(\mathbf{v})) \quad \text { with } \boldsymbol{\alpha} \in \mathbb{R}^{\# N}
$$

where the Lagrange multipliers $\boldsymbol{\alpha}_{i} \in \mathbb{R}$ are uniquely determined by the moments constraints $\boldsymbol{\mu}^{K}(G)=\boldsymbol{\mu}^{K}(f)$.

Proof. We first recall the following result due to Junk [8]: let $f$ be a nonnegative function in $L^{1}(K), f \neq 0$ and set $\rho=\boldsymbol{\mu}^{K}(f)$ (remark that any moment of $f$ of finite order is defined if $f \in L^{1}(K)$ since $K$ is bounded) then there exists a unique $\boldsymbol{\alpha} \in \mathbb{R}^{\# N}$ such that $\rho=\boldsymbol{\mu}^{K}(\exp (\boldsymbol{\alpha} \cdot \mathbf{m}(\mathbf{v})))$. Junk's proof relies on the study of the continuous and differentiable function:

$$
\boldsymbol{\alpha} \longrightarrow z(\boldsymbol{\alpha})=\int_{K} \exp (\boldsymbol{\alpha} \cdot \mathbf{m}(\mathbf{v})) \mathrm{d} \mathbf{v}-\boldsymbol{\alpha} \cdot \rho .
$$

It is clear that this function is strictly convex due to the positive definiteness of its Hessian:

$$
\int_{K} \mathbf{m}(\mathbf{v}) \otimes \mathbf{m}(\mathbf{v}) \exp (\boldsymbol{\alpha} \cdot \mathbf{m}(\mathbf{v})) \mathrm{d} \mathbf{v}
$$

So if $z(\boldsymbol{\alpha})$ possesses a minimum then it is unique and it satisfies to

$$
\nabla_{\boldsymbol{\alpha}} z(\boldsymbol{\alpha})=0 \Longleftrightarrow \int_{K} \mathbf{m}(\mathbf{v}) \exp (\boldsymbol{\alpha} \cdot \mathbf{m}(\mathbf{v})) \mathrm{d} \mathbf{v}=\rho
$$

The existence of such a minimum is then just the question of proving the coercivity of $z(\boldsymbol{\alpha})$. So let us prove that

$$
\lim _{|\boldsymbol{\alpha}| \rightarrow+\infty} z(\boldsymbol{\alpha})=+\infty \text { or equivalently } \forall \boldsymbol{\alpha} \neq 0 \lim _{s \rightarrow+\infty} z(s \boldsymbol{\alpha})=+\infty
$$

Let $\boldsymbol{\alpha} \neq 0$ and suppose firstly that $\boldsymbol{\alpha} \cdot \mathbf{m}(\mathbf{v}) \leq 0$ on $K$ then

$$
\boldsymbol{\alpha} . \rho=\boldsymbol{\alpha} \cdot \boldsymbol{\mu}^{K}(f)=\langle\boldsymbol{\alpha} \cdot \mathbf{m}(\mathbf{v}) f(\mathbf{v})\rangle<0
$$

This strict inequality holds because on one hand $\boldsymbol{\alpha} \cdot \mathbf{m}(\mathbf{v})<0$ except at a finite number of roots and on the other hand $f \neq 0$ means that $f>0$ in a set $\omega \subset K$ with $|\omega| \neq 0$. Next remark that $z(s \boldsymbol{\alpha})>-s \boldsymbol{\alpha} . \rho$ and consequently $z(s \boldsymbol{\alpha}) \rightarrow+\infty$ as $s \rightarrow+\infty$.

In the second case there exists a non-empty set $\omega$ and $\epsilon>0$ such that $\boldsymbol{\alpha} \cdot \mathbf{m}(\mathbf{v}) \geq \epsilon$ for all $\mathbf{v} \in \omega$. Then

$$
z(s \boldsymbol{\alpha}) \geq \int_{\omega} \exp (s \boldsymbol{\alpha} \cdot \mathbf{m}(\mathbf{v}))-s \boldsymbol{\alpha} \cdot \rho \geq|\omega| \exp (s \epsilon)-s \boldsymbol{\alpha} . \rho \rightarrow+\infty \text { as } s \rightarrow+\infty .
$$

The second part of the proof consists in showing that $G(\mathbf{v})=\exp (\boldsymbol{\alpha} \cdot \mathbf{m}(\mathbf{v}))$ - where $\boldsymbol{\alpha}$ defined above - is the unique minimizer of $H_{K}$ on the set of constraints $\boldsymbol{\mu}^{K}(g)=\rho$. Remark that there exists positive $m$ and $M$ such that $m \leq G(\mathbf{v}) \leq M$ on $K$. Consequently $H_{K}$ is Gateaux-differentiable at $G$ in $L^{\infty}(K)$ and

$$
H_{K}^{\prime}(G) \cdot \varphi=\int_{K} \varphi \ln G \mathrm{~d} \mathbf{v}=\boldsymbol{\alpha} \cdot \boldsymbol{\mu}^{K}(\varphi), \quad \forall \varphi \in L^{\infty}(K)
$$

Now for any nonnegative function $g \in L^{1}(K)$ such that $\boldsymbol{\mu}^{K}(g)=\rho$ we define $g_{n}$ as

$$
g_{n}(\mathbf{v})=\min (g(\mathbf{v}), n)
$$

Up to the extraction of a subsequence $g_{n} \rightarrow g$ a.e and in $L^{1}(K)$ (dominated convergence) and $\boldsymbol{\mu}^{K}\left(g_{n}\right) \rightarrow$ $\boldsymbol{\mu}^{K}(g)=\rho$. One has also

$$
g_{n} \ln g_{n}-g_{n} \longrightarrow g \ln g-g \text { a.e. }
$$


since $x \rightarrow x \ln x-x$ is continuous while on the other hand

$$
\left|g_{n} \ln g_{n}-g_{n}\right| \leq g(|\ln g|+1), \quad \forall n \geq 1 .
$$

According to Lemma 1 this last function is in $L^{1}(K)$ provided $H(g)<+\infty$. So using again dominated convergence $H\left(g_{n}\right) \rightarrow H(g)$. The convexity of $H$ and (6) implies that

$$
H_{K}\left(g_{n}\right)-H_{K}(G) \geq H_{K}^{\prime}(G) \cdot\left(g_{n}-G\right)=\boldsymbol{\alpha} \cdot \boldsymbol{\mu}^{K}\left(g_{n}-G\right) .
$$

Passing to the limit on both sides gives

$$
H_{K}(g)-H_{K}(G) \geq 0
$$

since $\boldsymbol{\mu}^{K}\left(g_{n}\right) \rightarrow \boldsymbol{\mu}^{K}(g)=\boldsymbol{\mu}^{K}(G)=\rho$. Hence $G$ is a global minimizer of $H_{K}$ under the constraints $\boldsymbol{\mu}^{K}(G)=\rho$ and it is unique thanks to the strict convexity of $H_{K}$.

Let us now finish the proof of Theorem 1:

Proof. Step 1. Existence of a minimizer $G$ in the set of constraints $C_{f}$

Let $g_{n}$ be a minimizing sequence:

$$
\lim H\left(g_{n}\right)=\inf _{g \in C_{f}} H(g) .
$$

From Lemma $1(3),\left(g_{n}\right)_{n}$ is weakly compact in $L^{1}$ so up to the extraction of a sub-sequence there exists $G \in L^{1}$ such that $g_{n} \rightarrow G$. Now let us remark that $C_{f}$ is strongly and weakly closed in $L^{1}$. Indeed if $\left(h_{n}\right)_{n} \rightarrow h$ in $L^{1}$ and $h_{n} \in C_{f}$ then

$$
\int h|\mathbf{v}|^{N} \mathrm{~d} \mathbf{v} \leq \liminf \int h_{n}|\mathbf{v}|^{N} \mathrm{~d} \mathbf{v}
$$

(Fatou lemma) and for any $R>0$ and $\mathbf{q} \in \mathbf{N}^{d},|\mathbf{q}| \leq N-1$ one has:

$$
\int_{|\mathbf{v}| \leq R} h \mathbf{v}^{\mathbf{q}} \mathrm{d} \mathbf{v}=\lim \int_{|\mathbf{v}| \leq R} h_{n} \mathbf{v}^{\mathbf{q}} \mathrm{d} \mathbf{v}=\int f \mathbf{v}^{\mathbf{q}} \mathrm{d} \mathbf{v}-\lim \int_{|\mathbf{v}|>R} h_{n} \mathbf{v}^{\mathbf{q}} \mathrm{d} \mathbf{v} .
$$

But $\int_{|\mathbf{v}|>R} h_{n} \mathbf{v}^{\mathbf{q}} \mathrm{d} \mathbf{v}$ is uniformly bounded by $\left\langle f|\mathbf{v}|^{N}\right\rangle / R$ so letting $R \rightarrow+\infty$, one obtains $\boldsymbol{\mu}(h) \leq^{*} \boldsymbol{\mu}(f)$ and $h \in C_{f}$ ! But $C_{f}$ is convex so that it is also weakly closed and consequently $G \in C_{f}$. Then $H$ is l.s.c in $L_{N}^{1}$ and $H$ is strictly convex on its domain imply that $G$ is the unique minimizer of $H$ on $C_{f}$.

Step 2. Characterization of $G$

$G$ is the unique solution to the minimization problem (5) implies that either the last constraint is qualified or it is not. Those two cases write:

(1) $\boldsymbol{\mu}(G)=\boldsymbol{\mu}(f)$;

(2) $\boldsymbol{\mu}(G) \leq^{*} \boldsymbol{\mu}(f)$ and $\left\langle G|\mathbf{v}|^{N}\right\rangle\left\langle\left\langle f|\mathbf{v}|^{N}\right\rangle\right.$. In that case the constraint of highest degree no longer plays a role to characterize $G$ as a consequence of the convexity of $H$.

We are going to prove in the two cases that there exists $m(\mathbf{v}) \in \mathbb{P}$ such that $G(\mathbf{v})=\exp (m(\mathbf{v})) \in C_{f}$. To do so we use the characterization of the entropy minimizer on a bounded set (Lem. 2). Let us consider the first case and set $\rho_{K}=\boldsymbol{\mu}^{K}(G)$ for a given compact set $K$. Using Lemma 2 there exists a unique solution $G_{K}$ to the minimization problem:

$$
\min \left\{H_{K}(g), \boldsymbol{\mu}^{K}(g)=\rho_{K}\right\} \cdot
$$

This solution writes $G_{K}(\mathbf{v})=\exp \left(\boldsymbol{\alpha}^{K} \cdot \mathbf{m}(\mathbf{v})\right)$ where $\boldsymbol{\alpha}^{K} \in \mathbf{R}^{\# P}$ is uniquely determined by the moments constraints $\boldsymbol{\mu}^{K}\left(G_{K}\right)=\rho_{K}$. We now want to prove that $G$ restricted to $K$ is $G_{K}$. It is quite simple by setting:

$$
\tilde{G}(\mathbf{v})=\left\{\begin{array}{l}
G_{K}(\mathbf{v}) \text { if } \mathbf{v} \in K \\
G(\mathbf{v}) \text { otherwise }
\end{array}\right.
$$


and remarking that $\boldsymbol{\mu}(\tilde{G})=\rho^{K}+\boldsymbol{\mu}^{C_{K}}(G)=\boldsymbol{\mu}(G)$ and

$$
H(\tilde{G})=H_{K}\left(G_{K}\right)+H_{C_{K}}(G) \leq H_{K}(G)+H_{C_{K}}(G)=H(G)
$$

$\left(C_{K}=\mathbb{R}^{d} / K\right)$ so that $\tilde{G} \in C_{f}$ and $\tilde{G}$ minimizes the entropy as well. Hence $\tilde{G}=G$ and there exists an implicit relation between the moments of $G$ on $K-\rho^{K}$ - and the coefficients $\boldsymbol{\alpha}^{K}$ :

$$
\boldsymbol{\mu}^{K}\left(\exp \left(\boldsymbol{\alpha}^{K} \cdot \mathbf{m}(\mathbf{v})\right)=\rho^{K} .\right.
$$

Let us now consider a sequence of compact sets $K_{n}-$ e.g. $K_{n}=B(0, n)$ - such that $K_{n} \subset K_{n+1}$ and $\cup_{n} K_{n}=\mathbb{R}^{d}$. We denote by $\boldsymbol{\alpha}^{n}$ the above coefficients for $K=K_{n}$. Those coefficients do not depend on $K_{n}$ since

$$
G(\mathbf{v})=\exp \left(\boldsymbol{\alpha}^{n} \cdot \mathbf{m}(\mathbf{v})\right)=\exp \left(\boldsymbol{\alpha}^{n+1} \cdot \mathbf{m}(\mathbf{v})\right) \forall \mathbf{v} \in K_{n} .
$$

Therefore for all $\mathbf{v} \in \mathbf{R}^{d}, G$ has the form $G(\mathbf{v})=\exp (\boldsymbol{\alpha} \cdot \mathbf{m}(\mathbf{v}))$ and

$$
\boldsymbol{\mu}^{K_{n}}\left(\exp (\boldsymbol{\alpha} \cdot \mathbf{m}(\mathbf{v}))=\rho^{K_{n}} .\right.
$$

Passing to the limit in $n$ in this last equation (dominated convergence in $L_{N}^{1}$ with the functions $f_{n}=\left.\exp (\boldsymbol{\alpha} \cdot \mathbf{m}(\mathbf{v}))\right|_{K_{n}}, 0$ otherwise) we obtain a relation that determines entirely the Lagrange multipliers:

$$
\boldsymbol{\mu}\left(\exp (\boldsymbol{\alpha} \cdot \mathbf{m}(\mathbf{v}))=\lim _{n \rightarrow \infty} \rho^{K_{n}}=\boldsymbol{\mu}(f)\right.
$$

(no further condition is required to ensure that $\boldsymbol{\mu}(G)=\boldsymbol{\mu}(f)$ ). The second case can be treated in a similar way by omitting the constraint on the moment of highest degree and leads to an exponential form where the Lagrange multiplier corresponding to that moment is null. So in the second case $G$ writes

$$
G(\mathbf{v})=\exp \left(\sum_{|\mathbf{q}| \leq N-1} \alpha_{\mathbf{q}} \mathbf{v}^{\mathbf{q}}\right)
$$

where the Lagrange multipliers $\alpha_{\mathbf{q}}$ are uniquely determined by the relations $\mu_{\mathbf{q}}(G)=\mu_{\mathbf{q}}(f)(|\mathbf{q}| \leq N-1)$. Remark however that when $N$ is even then all $\alpha_{\mathbf{q}}$ with $|\mathbf{q}|=N-1$ must vanish since otherwise $\langle G\rangle$ would diverge. This means that in that case the corresponding moments of order $N-1$ play no role in determining the other Lagrange multipliers $\alpha_{\mathbf{q}}(\mathbf{q} \leq N-2)$ and only the constraints of degree less than $N-1$ are required to determine $G$. This is a particular case where the moments of order $N-1$ depend on those of less order.

Step 3. Counterpart

Suppose now that there exists $G \in C_{f}$ with $G(\mathbf{v})=\exp (\boldsymbol{\alpha} \cdot \mathbf{m}(\mathbf{v})) \in \exp (\mathbf{m})$. We proceed as in Lemma 2 and remark that $H$ is Gateaux-differentiable at $G$ in

$$
L_{G}^{\infty}\left(\mathbb{R}^{d}\right)=\left\{\varphi \text { such that } \frac{\varphi}{G} \in L^{\infty}\left(\mathbf{R}^{d}\right)\right\}
$$

and one has

$$
\forall \varphi \in L_{G}^{\infty}\left(\mathbf{R}^{d}\right): \quad H^{\prime}(G) \cdot \varphi=\boldsymbol{\alpha} \cdot \boldsymbol{\mu}(\varphi) .
$$

Then using the convexity of $H$ one obtains

$$
\forall \varphi \in L_{G}^{\infty}, \varphi \geq 0: \quad H(G)-H(\varphi) \leq H^{\prime}(G) .(G-\varphi)=\boldsymbol{\alpha} \cdot \boldsymbol{\mu}(G-\varphi) .
$$


Next we want to prove that $H(G) \leq H(g)$ for all $g \in C_{f}$. Let $g \in C_{f}$ with $H(g)<\infty$ (we can always assume that this holds otherwise $H(G) \leq \bar{H}(g)$ is obvious). We define $g_{n}$ as

$$
g_{n}(\mathbf{v})=\min (g(\mathbf{v}), n) \text { if }|\mathbf{v}| \leq n, \quad 0 \text { otherwise. }
$$

One can proceed exactly as in Lemma 2 to prove that up to the extraction of a subsequence on has on one hand $g_{n} \rightarrow g$ in $L_{N}^{1}\left(\Rightarrow \boldsymbol{\mu}\left(g_{n}\right) \rightarrow \boldsymbol{\mu}(g)\right)$ and on the other hand $H\left(g_{n}\right) \rightarrow H(g)$. Indeed one can apply the dominated convergence theorem on $h_{n}=g_{n} \ln g_{n}-g_{n}$ to prove that $h_{n} \rightarrow g(\ln g-1)$ in $L^{1}$. Taking $\varphi=g_{n}$ in $(8)$ and passing to the limit yields

$$
\forall g \in C_{f}, \quad H(G)-H(g) \leq \boldsymbol{\alpha} \cdot \boldsymbol{\mu}(G-g) .
$$

But the right hand side reduces to $\alpha_{\# P}\left\langle(G-g)|\mathbf{v}|^{N}\right\rangle$ since $g$ and $G$ are in $C_{f}$. Then either $\alpha_{\# P}=0$ (constraint of highest degree not qualified) or $\alpha_{\# P}<0$ (due to $\langle G\rangle\left\langle\infty\right.$ ) and $\left\langle g|\mathbf{v}|^{N}\right\rangle \leq\left\langle G|\mathbf{v}|^{N}\right\rangle$. In both cases the right hand side is $\leq 0$ which implies that $H(G)-H(g) \leq 0 \forall g \in C_{f}$ and $G$ is the entropy minimizer in $C_{f}$.

Finally remark that in the case where the last constraint is not qualified one can proceed the same way to prove that

$$
H(G)-H(g) \leq 0 \forall g \in\left(L_{N-1}^{1}\right)^{+}, \quad \mu_{\mathbf{q}}(g)=\mu_{\mathbf{q}}(f),|\mathbf{q}| \leq N-1
$$

that is $G$ is a global minimizer of $H$ in $L_{N-1}^{1}$ under the above constraints.

Remark 1. When $N=2$, Theorem 1 implies that the unique minimizer of the entropy in

$$
C=\left\{g \geq 0,\langle g\rangle=\rho_{0},\langle g \mathbf{v}\rangle=\rho_{1},\left\langle g|\mathbf{v}|^{2}\right\rangle \leq \rho_{2}\right\}
$$

where $\rho=\left\langle f\left(1, \mathbf{v},|\mathbf{v}|^{2}\right)^{T}\right\rangle$ for some nonnegative function $f \in L_{2}^{1}\left(\mathbb{R}^{d}\right)$ qualifies all constraints. This unique minimizer is the well-known Maxwellian distribution (13) whose parameters are entirely determined by the mass, momentum and energy of $f$.

Remark 2. Csiszar introduced in 1973 the concept of I-projection which he defines as follows. Let $P, Q$ be two probability distributions and define the relative entropy (or Kullback-Leibler information) as:

$$
I(P \mid Q)=\int \log p_{Q} \mathrm{~d} P=\left\{\begin{array}{l}
\int p_{Q} \log p_{Q} \mathrm{~d} Q \text { if } P \ll Q \\
+\infty \text { otherwise }
\end{array}\right.
$$

where $P \ll Q$ means " $P$ absolutely continuous with respect to $Q$ " and $p_{Q}$ is the Radon-Nikodym derivative. We may redefine this relative entropy for two $L^{1}$ nonnegative functions of same mass as:

$$
I(f \mid g)=\left\{\begin{array}{l}
\int f \log \frac{f}{g} \text { if } f \ll g \\
+\infty \text { otherwise }
\end{array}\right.
$$

where here $f \ll g$ essentially means that when $g$ vanishes then so does $f$. Then he proves that given a closed convex set $\mathcal{E}$ in $L^{1}$ (variation closed in his terminology) there exists always a solution to the minimization problem

$$
I(q \mid r)=\min _{g \in \mathcal{E}} I(g \mid r)
$$

which he calls the I-projection of $r$ onto $\mathcal{E}$. Remark first that

$$
I(g \mid r)=H(g)-\int g \ln r
$$


and that $\langle g \ln r\rangle=$ constant depending only on $\mathcal{E}$ if $\mathcal{E}=C_{f}$ defined in Theorem 1 and if $r(\mathbf{v})=\exp (m(\mathbf{v}))$ with $m(\mathbf{v}) \in \mathbb{P}_{N-1}$. This means that in this particular case the two minimization problems (5) and (9) are equivalent and consequently the I-projection on $C_{f}$ of any $\exp \left(\mathbb{P}_{N-1}\right)$-function coincides with the entropic approximation of $f$ on $\exp (\mathbb{P})$.

The assumption "E्E closed" amounts in our case to relaxing the last constraint. Remark also that in his proof the existence of a converging minimizing sequence is obtained by using the celebrated Csiszar-Kullback inequality:

$$
\|f-g\|_{L^{1}}^{2} \leq 2 I(f \mid g) .
$$

In his case it is the relative entropy that gives the control on such a sequence while it is the entropy in our case together with Dunford-Pettis criteria. This does not make much difference.

The main difference between his proof and ours is the characterization of the I-projection. He essentially proves that if $G$ exists and qualifies all constraints in a set of linear constraint then $G$ must take the exponential form. It is clear that this would be sufficient if all constraints would be qualified - for example in the case of bounded domains of integration (see Lem. 2). This is also the case when the set of constraints is spanned by some $(\phi)_{i}$ 's with $\phi_{i}$ odd except for one $\phi_{N}$ which is even and controls any other $\phi_{i}$ at infinity. So in this sense our theorem enlarges the spectrum of application of the I-projection.

Remark 3. The result of Theorem 1 can be extended to the case of nonnegative function in $L_{N}^{1}$ with unbounded entropy. Indeed a result due to Junk [9] sets that for such function there exists a function $g$ compactly supported and bounded such that $\boldsymbol{\mu}(g)=M u(f)$. This ensures the existence of a minimizing sequence of $H$ in $C_{f}$ !

\section{Application to Levermore's moment ClOsure in Kinetic theory}

Our first application of Theorem 1 concerns Levermore's approach in kinetic theory [11]. Let us first recall his framework. Suppose we want to solve the kinetic equation

$$
\partial_{t} f+\mathbf{v} \cdot \nabla f=C(f)
$$

where $f=f(t, \mathbf{x}, \mathbf{v})$ is the density function $(t, \mathbf{x}, \mathbf{v}) \in \mathbb{R}^{+} \times \mathbb{R}^{d} \times \mathbb{R}^{d}$ and $C(f)$ is a collision operator acting only on the $\mathbf{v}$ dependence of $f$. The above equation describes the behavior of a gas where particles evolve according to their own velocity and through the interaction with other particles. A particularly important equation of that form is the Boltzmann equation which will be discussed in the next section. At the moment let us just assume that $C(f)$ satisfies the following quite general and physically meaningful properties:

(1) Conservation laws: for every $f \in D(C)$ - the domain of definition of $C$ which is assumed to be contained in the cone of nonnegative functions - one has

$$
\langle C(f) \varphi\rangle=0, \quad \forall \varphi \in \mathbb{E}_{0}=\operatorname{span}\left[1, v_{1}, v_{2}, \ldots, v_{d},|\mathbf{v}|^{2}\right] .
$$

(2) Dissipation of entropy:

$$
\forall f \in D(C) \quad\langle\ln f C(f)\rangle \leq 0
$$

with equality if and only if:

$$
f(\mathbf{v})=M(\mathbf{v}) \equiv \frac{\rho}{(2 \pi T)^{d / 2}} \exp \left(-\frac{(\mathbf{v}-\mathbf{u})^{2}}{2 T}\right),
$$

for some $(\rho, \mathbf{u}, T) \in \mathbb{R}^{+} \times \mathbb{R}^{d} \times \mathbb{R}^{+}$. 
(3) Galilean invariance: let $\tau_{\mathbf{u}}$ define the translation operator $\tau_{\mathbf{u}} f(\mathbf{v})=f(\mathbf{v}-\mathbf{u})$ and for any $O \in S O\left(\mathbb{R}^{d}\right)$ (the group of orthogonal transformations in $\mathbb{R}^{d}$ ) define $\tau_{O}$ as $\tau_{O} f(\mathbf{v})=f(O \mathbf{v})$. Then

$$
\tau_{u} C(f)=C\left(\tau_{\mathbf{u}} f\right), \quad \tau_{O} C(f)=C \tau_{O}(f) .
$$

Levermore's approach can be seen as a Galerkin method for solving (10). It is performed in two steps: the first one is rather standard and consists in projecting equation (10) onto a given polynomial space $\mathbf{m}$. Using the notations of section 1 this first step reads

$$
\partial_{t}\langle\mathbf{m}(\mathbf{v}) f\rangle+\nabla \cdot\langle\mathbf{v m}(\mathbf{v}) f\rangle=\langle\mathbf{m}(\mathbf{v}) C(f)\rangle \cdot
$$

This system of \#P-moments equations is of course not closed since fluxes of highest order are not defined. Then the second step consists in a non-standard way of projecting $f$ : first onesolves the minimization problem

$$
\min \{H(g),\langle\mathbf{m}(\mathbf{v}) g\rangle=\langle\mathbf{m}(\mathbf{v}) f\rangle\}
$$

and secondly one remarks that according to Section 3 the solution should belong to $\exp (\mathbb{P})$. That is the non-linear projection of $f$ is of the form $\exp (\boldsymbol{\alpha} \cdot \mathbf{m}(\mathbf{v}))$ and the complete approximation of (10) reads:

Find $\boldsymbol{\alpha}(t, \mathbf{x})$ solution to the system of equations:

$$
\partial_{t}\langle\mathbf{m}(\mathbf{v}) \exp (\boldsymbol{\alpha} \cdot \mathbf{m}(\mathbf{v}))\rangle+\nabla \cdot\langle\mathbf{v m}(\mathbf{v}) \exp (\boldsymbol{\alpha} \cdot \mathbf{m}(\mathbf{v}))\rangle=\langle\mathbf{m}(\mathbf{v}) C(\exp (\boldsymbol{\alpha} \cdot \mathbf{m}(\mathbf{v})))\rangle \cdot
$$

Another (and more physical) way to interpret that second step is to speak about "closure relation". Fluxes of highest order are considered as functions of moments of lower order - so-called closure relations - through the assumption that the density function $f$ is closed to a local equilibrium function. The most classical example is to consider $f$ as a local Maxwellian distribution; the closure relation is then nothing but the second law of thermodynamic and the above system of equations is the Euler system of equations.

Remark that performing the change of variable $f \rightarrow f^{*}=\ln f$ in (10) and then projecting both the equation and $f^{*}$ on $\mathbf{m}$ leads to the above system of equation. This justifies once more the term "Galerkin approach" but again the projection of $f^{*}$ is absolutely non-standard. All the theory leans on the fact that $f^{*}$ is the polar variable of $f$ with respect to the strictly convex function $h(f)=f \ln f-f$. At a macroscopic level - that is at the level of system (15) - this reads $\boldsymbol{\alpha}$ is the polar variable of the moments $\rho$ with respect to the entropy

$$
h(\rho)=\min _{g}\{H(g),\langle\mathbf{m}(\mathbf{v}) g\rangle=\rho\}
$$

All features concerning system (15) such as hyperbolicity or entropy dissipation can be derived from this supposed one-to-one relation between $\boldsymbol{\alpha}$ and $\boldsymbol{\rho}$. But this relation raises many questions related to Section 3 . The existence of a solution is indeed subjected to the restriction that the hyperbolic system (15) should produce moments $\boldsymbol{\rho}$ that can be represented by exponential functions. Those exponential functions are precisely solutions to minimization problems such as (16). The problem is that Theorem 1 just ensures the existence of a single solution in a set of constraints where the one of highest order is relaxed. So the first question that arises is what is the set of moments for which all constraints are qualified? We may call this set the domain of definition $D(h)$ of the entropy $h$. Then is $D(h)$ equivalent to the set of moments $\boldsymbol{\mu}\left(\left(L_{N}^{1}\right)^{+}\right)$(the set of moments of all nonnegative $L_{N}^{1}$-functions)? This is called a realizability problem. Such a problem has already been addressed by Junk in two different papers. In [8] he gives a complete description of $D(h)$ in the particular case of "Levermore's five-moment system" while in [9] he essentially proves that $D(h)$ is the set of moments of integrable exponential functions (with polynomial exponent of the form $\boldsymbol{\alpha} \cdot \mathbf{m}(\mathbf{v})$ ). Here we prove that his results can be derived directly from Theorem 1 . 
Levermore proposes to set the system of equations (15) for particular spaces of approximation P. More precisely such spaces are assumed to fulfill the following requirements:

(I) $\mathbb{E}_{0}=\operatorname{span}\left[1, v_{1}, v_{2}, \ldots, v_{d},|\mathbf{v}|^{2}\right] \subset \mathbf{P}$.

(II) $\mathbf{P}$ is invariant under the actions of $\tau_{\mathbf{u}}$ and $\tau_{O}$.

(III) The cone $\mathbf{P}_{c}=\{m \in \mathbf{P}:\langle\exp (\mathbf{m}(\mathbf{v}))\rangle<\infty\}$ has nonempty interior in $\mathbf{P}$.

Property (I) allows to recover conservation laws (11). (II) is set to ensure Galilean invariance of the whole theory. (III) is a necessary (but not sufficient) condition for the system (15) to be well-posed. The problem is that system (15) can produce moments in $\boldsymbol{\mu}\left(\left(L_{N}^{1}\right)^{+}\right)$that might not fall into $\boldsymbol{\mu}\left(\exp \left(\mathbb{P}_{c}\right)\right)$. We call this a realizability problem. Our concern here is to describe properly the domain for which this problem occurs.

Remark first that it is quite easy to satisfy (I) and (II) if $\mathbf{P}$ is polynomial so that we can assume from now on that $\mathbf{P}$ is contained within a certain $\mathbf{P}_{N}$. Next condition (III) implies that $N=\operatorname{deg} \mathbf{P}$ must be even and that there are monomials of degree $N$ of the form $\mathbf{v}^{2 \mathbf{q}}$ (with $\mathbf{q} \in \mathbb{N}^{d}$ and $2|\mathbf{q}|=N$ ) that can control any other monomials of $\mathbf{P}$ at infinity. We shall still denote with $\leq^{*}$ the relations which here have the general meaning:

$$
\boldsymbol{\mu}(g) \leq^{*} \boldsymbol{\mu}(f) \Longleftrightarrow \boldsymbol{\mu}(g) \in \boldsymbol{\mu}\left(\bar{C}^{L^{1}}\right)
$$

where $\bar{C}^{L^{1}}$ is the closure of $C=\{g \geq 0 / \boldsymbol{\mu}(g)=\boldsymbol{\mu}(f)\}$ in $L^{1}$. It is clear that this definition amounts to the previous one when

$$
(\mathrm{III})^{\prime} \mathbf{P}=\mathbb{E} \oplus \mathbb{R}|\mathbf{v}|^{N} \quad \text { with } \mathbb{E} \subset \mathbf{P}_{N-1}
$$

since then

$$
\begin{gathered}
g_{n} \rightarrow g \text { in } L^{1} \text { and } g_{n} \in\left(L_{N}^{1}\right)^{+} \Longrightarrow \\
\left\langle g \mathbf{v}^{\mathbf{q}}\right\rangle=\lim \left\langle g_{n} \mathbf{v}^{\mathbf{q}}\right\rangle, \quad|\mathbf{v}| \leq N-1 \text { and }\left\langle g|\mathbf{v}|^{N}\right\rangle \leq \liminf \left\langle g_{n}|\mathbf{v}|^{N}\right\rangle .
\end{gathered}
$$

Then our general result implies the following theorem:

Theorem 2. Let $\mathbf{P}$ be a polynomial space that satisfies (I),(II),(III) and $\boldsymbol{\rho} \in \mathbb{R}^{\# P}$. Then $\boldsymbol{\rho}$ is realizable in the sense that "there exists $m(\mathbf{v}) \in \mathbf{P}$ solution to $\boldsymbol{\mu}(\exp (m(\mathbf{v})))=\boldsymbol{\rho}$ " if and only if:

(i) $\boldsymbol{\rho} \in \boldsymbol{\mu}\left(\left(L_{N}^{1}\right)^{+}\right)$;

(ii) there exists no function in $\exp (\mathbf{P})$ contained in $\bar{C}^{L^{1}} / C$ where $\bar{C}^{L^{1}}$ is the $L^{1}$-closure of

$$
C=\{g \geq 0 \text { a.e., } \boldsymbol{\mu}(g)=\boldsymbol{\rho}\}
$$

Proof. The condition $\boldsymbol{\rho} \in \boldsymbol{\mu}\left(\left(L_{N}^{1}\right)^{+}\right)$is necessary and sufficient to ensure the existence of a minimizer of $H$ in $C$ according to Theorem 1 . The only thing to check is that Theorem 1 still applies in the general case of space $\mathbf{P}$ satisfying (I),(II),(III). Remark first that Lemmas 1, 2 and 3 are still valid. Next a minimizing sequence of $H$ in $C=\{g \geq 0, \boldsymbol{\mu}(g)=\boldsymbol{\mu}(f)\}$ only converges in its closure in $L^{1}-\bar{C}^{L^{1}}-$ and its limit $G$ takes the exponential form $G(\mathbf{v})=\exp (m(\mathbf{v}))$ with $m(\mathbf{v}) \in \mathbb{P}$. The important point is the uniqueness of this solution: it is not only the unique solution to the minimization problem in $C$, it is also the unique solution in $\exp (\mathbb{P})$ to the problem $\boldsymbol{\mu}\left(\exp (m(\mathbf{v})) \leq^{*} \boldsymbol{\rho}\right.$ according to the counterpart of Theorem 1 . Then either $\boldsymbol{\mu}(G)=\boldsymbol{\rho}$ or $\boldsymbol{\mu}(G) \leq^{*} \boldsymbol{\rho}$ with strict inequality for at least one component in which case $\rho$ is not realizable. 
Remark 4. Junk proceeds in [9] as follows: he first considers formally the problem of minimization and concludes that the exponential form of the minimizer is a necessary condition. Then he deduces from the shape of the set

$$
\Lambda=\left\{\lambda \in \mathbb{R}^{\# P} /\langle\exp (\lambda \cdot \mathbf{m}(\mathbf{v})\rangle<\infty\}\right.
$$

sufficient conditions for the existence of the minimizer. His approach considers Lagrange multipliers and is a duality argument while ours is a direct and rather classical one.

Corollary 1. All exponential functions are entropy minimizers under suitable constraints. More precisely let $G(\mathbf{v})=\exp (m(\mathbf{v}))$ with $m(\mathbf{v})$ polynomial and let $\mathbf{P}$ be any polynomial space containing $m$ and satisfying (I),(II),(III) then $G$ is the unique minimizer of the entropy in all sets of the form

$$
C=\left\{g \geq 0, \boldsymbol{\mu}(g) \mathbf{x}^{*} \boldsymbol{\rho}\right\}
$$

where $\boldsymbol{\rho} \in \mathbb{R}^{\# P}$ just satisfies $\boldsymbol{\rho} \geq^{*} \boldsymbol{\mu}(G)$.

Example 1. As an application of Theorem 2 let us study the problem already solved by Junk in [8] concerning the domain of definition of Levermore's five-moment system. Let $d=1$ and $\operatorname{set} \mathbf{P}=\operatorname{span}\left[1, v, v^{2}, v^{3}, v^{4}\right]$. We are interested in defining the set of moments $\rho \in \mathbb{R}^{5}$ for which the minimization problem

$$
\min \{H(g), \boldsymbol{\mu}(g)=\boldsymbol{\rho}\}
$$

possesses a solution. A necessary condition for this problem to possess a solution is $C \neq \emptyset$. According to Junk (see [8] and references therein) such a property relies on the positive definiteness of the Hankel matrix $\left(\rho_{i+j}\right)_{i, j=0,1,2}$. For example in the case of normalized mass, momentum and energy

$$
\overline{\boldsymbol{\rho}}=\left(1,0,1, \rho_{3}, \rho_{4}\right)^{T} \in \boldsymbol{\mu}\left(\left(L_{4}^{1}(\mathbb{R})\right)^{+}\right) \Longleftrightarrow \rho_{4}>1+\rho_{3}^{2}
$$

Suppose that $\boldsymbol{\rho}$ fulfills that condition then there exists a solution $G \in \partial C$ to the problem $\min _{g \in C} H(g)$ iff there exists no function of $\exp \left(\mathbf{P}_{c}\right)$ in the interior of $\bar{C}^{L^{1}}$. Such functions necessarily take the form $\tilde{G}(\mathbf{v})=$ $\exp \left(\alpha_{0}+\alpha_{1} v+\alpha_{2} v^{2}\right)$ (see Th. 1). If such a $\tilde{G}$ exists then the first three relations determine entirely the Lagrange multipliers $\alpha_{i}$ :

$$
\tilde{G}(v)=\frac{\rho}{\sqrt{2 \pi T}} \exp \left(-\frac{(v-u)^{2}}{2 T}\right) \text { where } \rho=\rho_{0}, u=\frac{\rho_{1}}{\rho_{0}}, T=\frac{\rho_{2}}{\rho_{0}}-\left(\frac{\rho_{1}}{\rho_{0}}\right)^{2}
$$

and its third and fourth moments are defined by the previous ones

$$
\tilde{\rho_{3}}=\rho u\left(u^{2}+3 T\right), \quad \tilde{\rho_{4}}=\rho\left(u^{4}+6 T u^{2}+3 T^{2}\right) .
$$

Therefore the existence result reads:

$$
\begin{gathered}
\text { "Let } \boldsymbol{\rho} \in \mathbb{R}^{5}, \boldsymbol{\rho} \in \boldsymbol{\mu}\left(\left(L_{4}^{1}(\mathbb{R})\right)^{+}\right) \text {then there exists a minimizer of the entropy in } C \text { iff } \\
\left(\rho, \rho_{1}, \rho_{2}, \rho_{3}, \rho_{4}\right)^{T} \nsupseteq^{*}\left(\rho, \rho_{1}, \rho_{2}, \rho u\left(u^{2}+3 T\right), \rho\left(u^{4}+6 T u^{2}+3 T^{2}\right)\right)^{T} "
\end{gathered}
$$

In particular for the normalized density this reads:

"Let $\rho_{3}, \rho_{4} \in \mathbb{R}$ then there exists a minimizer of the entropy in $C=\left\{g \geq 0, \boldsymbol{\mu}(g)=\left(1,0,1, \rho_{3}, \rho_{4}\right\}\right.$ iff $\rho_{4}>1+\rho_{3}^{2}$ and $\left(\rho_{3}, \rho_{4}\right) \geq^{*}(0,3)$." 
Example 2. A second interesting example is the one where $d=3$ and

$$
\mathbb{P}=\operatorname{span}\left[1, \mathbf{v}, \mathbf{v} \wedge \mathbf{v},|\mathbf{v}|^{2} \mathbf{v},|\mathbf{v}|^{4}\right]
$$

since this is the minimal space that satisfies (I),(II),(III) (or (III)') and that contains all physically meaningful information: mass, momentum, stress tensor and heat flux. Let $f \in\left(L_{4}^{1}\left(\mathbb{R}^{3}\right)\right)^{+}$and let us denote by

$$
\begin{array}{r}
\rho=\langle f\rangle, \rho \mathbf{u}=\langle f \mathbf{v}\rangle, \Sigma=\langle f(\mathbf{v}-\mathbf{u}) \wedge(\mathbf{v}-\mathbf{u})\rangle, \mathbf{q}=\frac{1}{2}\left\langle f|\mathbf{v}-\mathbf{u}|^{2}(\mathbf{v}-\mathbf{u})\right\rangle \\
\rho_{4}=\left\langle f(\mathbf{v})|\mathbf{v}|^{4}\right\rangle
\end{array}
$$

its moments (remark here that we have expressed moments up to order 3 in terms of the physically relevant ones and that it is equivalent to give $\left.\left\langle f\left(1, \mathbf{v}, \mathbf{v} \wedge \mathbf{v},|\mathbf{v}|^{2} \mathbf{v}\right)^{T}\right\rangle\right)$. Then Theorem 2 tells us that either the constraint on the fourth order moment is qualified for the entropy minimizer $G$, or $G$ must takes the form $\exp (m(\mathbf{v}))$ with $m \in \operatorname{span}[1, \mathbf{v}, \mathbf{v} \wedge \mathbf{v}]$. In that case the constraints

$$
\langle G\rangle=\rho,\langle G \mathbf{v}\rangle=\rho \mathbf{u},\langle G(\mathbf{v}-\mathbf{u}) \wedge(\mathbf{v}-\mathbf{u})\rangle=\Sigma
$$

determine entirely $G$ :

$$
G(\mathbf{v})=\frac{\rho}{\pi^{3 / 2}} \sqrt{\operatorname{det} A} \exp \left(-(\mathbf{v}-\mathbf{u})^{T} \cdot A \cdot(\mathbf{v}-\mathbf{u})\right) \quad \text { with } A=\frac{\rho}{2} \Sigma^{-1}
$$

(remark that $A$ is well defined since $\Sigma$ is a symmetric definite positive matrix). Hence it is easy to see that the heat flux $\mathbf{q}$ is null while $\tilde{\rho}_{4}=\left\langle G(\mathbf{v})|\mathbf{v}|^{4}\right\rangle$ can be computed analytically. Denoting by $p=1 / 3 \operatorname{Tr} \Sigma$ the pressure and with $T=\rho^{-1} p$ the temperature of both $f$ and $G$, one obtains the relation

$$
\tilde{\rho}_{4}=\rho u^{4}+6 \rho u^{2} T+4 \mathbf{u}^{T} \cdot \Sigma \cdot \mathbf{u}+9 \rho T^{2}+\frac{2}{\rho} \operatorname{Tr}\left(\Sigma^{2}\right) .
$$

Remark that the fourth order moment of the Maxwellian distribution (13) is obtained by letting $\Sigma=p \mathcal{I} \mathcal{I}$ and gives

$$
\left\langle M(\mathbf{v})|\mathbf{v}|^{4}\right\rangle=\rho u^{4}+10 \rho u^{2} T+15 \rho T^{2}
$$

"Let $\rho, \rho \mathbf{u} u, \Sigma, \mathbf{q}$ and $\rho_{4}$ be the mass, momentum, energy, heat flux and fourth order moments of a nonnegative function then there exists a minimizer of the entropy in

$$
\begin{gathered}
C=\{g \geq 0:\langle g\rangle \rho,\langle g(\mathbf{v}-\mathbf{u})\rangle=\mathbf{0},\langle g(\mathbf{v}-\mathbf{u}) \wedge(\mathbf{v}-\mathbf{u})\rangle=\Sigma, \\
\left.\qquad \frac{1}{2}\left\langle g|\mathbf{v}-\mathbf{u}|^{2}(\mathbf{v}-\mathbf{u})\right\rangle=\mathbf{q},\left\langle g|\mathbf{v}|^{4}\right\rangle=\rho_{4}\right\} \\
\text { if and only if }\left(\mathbf{q}, \rho_{4}\right) \nsupseteq^{*}\left(\mathbf{0}, \rho_{4}\right) \text { with } \tilde{\rho}_{4} \text { defined above." }
\end{gathered}
$$

That is the problem of non qualified constraint may arise only for null heat flux functions.

\section{Generalized BGK models}

A second important application of the entropic approximation is the simplification of the Boltzmann collision operator. Let us recall that this operator reads

$$
\begin{gathered}
C(f)=Q(f, f)(\mathbf{v})=\int_{S^{2}} \int_{\mathbb{R}^{3}}\left(f^{\prime} f_{*}^{\prime}-f f_{*}\right) q\left(\mathbf{v}_{\star}-\mathbf{v}, \omega\right) \mathrm{d} \mathbf{v}_{\star} \mathrm{d} \omega, \\
\mathbf{g}=\mathbf{v}_{\star}-\mathbf{v}, \mathbf{v}^{\prime}=\mathbf{v}+(\mathbf{g}, \omega) \omega, \mathbf{v}_{\star}^{\prime}=\mathbf{v}_{\star}-(\mathbf{g}, \omega) \omega,
\end{gathered}
$$


where $f=f(t, \mathbf{x}, \mathbf{v})$ is the density function $\left(f_{*}=f\left(t, \mathbf{x}, \mathbf{v}_{\star}\right), f^{\prime}=f\left(t, \mathbf{x}, \mathbf{v}^{\prime}\right), f_{*}^{\prime}=f\left(t, \mathbf{x}, \mathbf{v}_{\star}^{\prime}\right)\right)$ and $q\left(\mathbf{v}_{\star}-\mathbf{v}, \omega\right)$ is the differential cross-section. A classical exercise consists in proving that $C(f)=Q(f, f)$ fulfils the conditions $(11),(12)$ with equality if and only if $f$ takes the form (13) and (14). That is the Boltzmann equation is mass, momentum and energy preserving, entropy dissipating and Galilean invariant. A second classical theory consists in studying the asymptotic fluid limit of the Boltzmann equation by expanding formally $f$ according to a small parameter $\epsilon$ which represents the rarefaction of the gas (Knudsen number):

$$
f_{\epsilon}=f_{0}+\epsilon f_{1}+\epsilon^{2} f_{2}+\ldots
$$

Then letting $\epsilon$ tend to 0 in the non-dimensional equation

$$
\partial_{t} f_{\epsilon}+\mathbf{v} \cdot \nabla f_{\epsilon}=\frac{1}{\epsilon} Q\left(f_{\epsilon}, f_{\epsilon}\right)
$$

leads through the so-called Chapman-Enskog analysis to a "hierarchy" of system of equations depending on the order of $\epsilon$. At the zeroth-order $f_{0}$ takes the Maxwellian form (13) (local equilibrium function) and it formally satisfies a kinetic equation whose first moments are precisely the Euler system of equations. The next order provides us with the Navier-Stokes equation with viscosity $\mu$ and thermal diffusivity $\kappa$ determined by the interaction potential. Those coefficients and in particular the so-called Prandtl number which expresses the ratio between viscosity and thermal diffusivity

$$
\operatorname{Pr}=\frac{\mu c_{p}}{\kappa}
$$

correspond to those of the true Navier-Stokes system and can even be used to determine the latter. This similarity occurs also for higher members in the hierarchy (Burnett, super Burnett, etc.) but the major input of this asymptotic theory is the correlation between the Boltzmann equation and the Euler and further on Navier-Stokes systems of equations.

Approximations or simplifications of the complicated collision operator $Q(f, f)$ should take more or less all those features into account. The simplest model that mimics most of those properties is the Bhatnagar, Gross and Krook (BGK) relaxation model:

$$
R(f)=\frac{1}{\tau}(M-f)
$$

where $M$ is the local equilibrium function defined in (13) whose first moments are those of $f$ :

$$
\rho=\langle f\rangle, \quad \rho \mathbf{u}=\langle f \mathbf{v}\rangle, \quad T=\frac{1}{3 \rho}\left\langle f|\mathbf{v}-\mathbf{u}|^{2}\right\rangle
$$

and $\tau$ is a relaxation time possibly depending on moments of $f$. All properties of the BGK model are derived from the characterization of $M$ :

" $M$ is the unique minimizer of the entropy in the set

$$
C=\left\{g \geq 0, \boldsymbol{\mu}_{0}(g)=\boldsymbol{\mu}_{0}(f)=\left\langle\left(1, \mathbf{v},|\mathbf{v}|^{2}\right)^{T} f\right\rangle\right\} "
$$

(see Th. 1 and Rem. 1). Indeed conservation of mass, momentum and energy (11) is a simple consequence of $M \in C_{f} \Rightarrow \boldsymbol{\mu}_{0}(M)=\boldsymbol{\mu}_{0}(f)$. The entropy dissipation law

$$
\langle R(f) \ln f\rangle \leq 0
$$

is easily deduced from the convexity of $H$ and the property $H(M) \leq H(f)$. Characterization of the equilibrium function for the BGK model relies on the same properties and (14) is straightforward. Unfortunately this model shows a deficiency when studying its asymptotic fluid limit. On one side the zeroth order still gives 
the Euler system of equations but the next member in the hierarchy (Navier-Stokes equations) is obtained with an incorrect Prandtl number $(=1)$. The reason for this is quite simple: relaxation rates for the moments corresponding to the pressure tensor and heat flux are not comparable for the two different models. It is however interesting to try to correct that deficiency especially in view of applications: the computation of the interaction term $R(f)$ is just a matter of computing $\left\langle f\left(1, \mathbf{v},|\mathbf{v}|^{2}\right)^{T}\right\rangle$ while computing $Q(f, f)$ at each velocity requires an integration on $S^{2} \times \mathbb{R}^{3}$. A correction is brought in the Ellipsoidal Statistical model (ES) so as to adjust the viscosity. In this model the following constraint is added in $C$ :

$$
\left.\int g(\mathbf{v}) B_{i j}(\mathbf{v}-\mathbf{u}) \mathrm{d} \mathbf{v}\right)_{i j}=t(\Sigma-p \mathcal{I} \mathcal{I}), \quad B_{i j}(\mathbf{v})=v_{i} v_{j}-\frac{|\mathbf{v}|^{2}}{3} \delta_{i j}
$$

where $\Sigma$ is the stress tensor of $f$ defined in (19). Then setting $t=1-1 / \operatorname{Pr}$ leads to the definition of a nonisotropic Gaussian function $G(21)$ which is such that at the asymptotic fluid limit the ratio between $\mu$ and $\kappa$ is correct.

Adding constraints in $C$ is the leading idea of our generalization of the BGK model. Notice that two conditions are required:

1 - The supplementary constraints must be such that they match the Boltzmann collision operator. This requires for example that the "new" relaxation model $R(f)$ gives a correct asymptotic fluid limit, or that $\langle R(f) m(\mathbf{v})\rangle=\langle Q(f, f) m(\mathbf{v})\rangle$ for given moments $m(\mathbf{v})$.

2 - The procedure must be entropy decaying.

As concerns the ES model, condition 1 is somehow fulfilled artificially: $G$ has no heat flux but the Prandtl number is correct. Therefore (moments of) $G$ does not lie between (moments of) $M$ and $f$ and the entropy decay for that model can be hardly seen as a direct consequence of the convexity of $H$ (see [1]). In this respect the fundamental difference between BGK and ES models is that on one side $M$ matches the positive part of the collision integral $Q^{+}(f, f)$ while $G$ does not. Indeed in the first case the set of constraints reads:

$$
C=\left\{g \geq 0, \boldsymbol{\mu}_{0}(g)=\rho^{-1}\left\langle\left(1, \mathbf{v},|\mathbf{v}|^{2}\right)^{T} Q^{+}(f, f)\right\rangle\right\}^{\prime}
$$

(where $\rho=\rho_{0}=\langle f\rangle$ ) so that

$$
\boldsymbol{\mu}_{0}(M)=\boldsymbol{\mu}_{0}\left(\rho^{-1} Q^{+}(f, f)\right)
$$

while the added constraint (25) cannot be written in terms of $Q^{+}(f, f)$ since:

$$
\left(\left\langle G v_{i} v_{j}\right\rangle\right)_{i j} \neq\left(\left\langle\rho^{-1} Q^{+}(f, f) v_{i} v_{j}\right\rangle\right)_{i j} .
$$

Therefore our generalization consists in adding constraints relative to $Q^{+}(f, f)$. For the sake of simplicity we shall consider the case of Maxwellian molecules for which:

$$
Q(f, f)(\mathbf{v})=Q^{+}(f, f)-\rho f \text { with } Q^{+}(f, f)=\int_{S^{2}} \int_{\mathbb{R}^{3}} f^{\prime} f_{*}^{\prime} \mathrm{d} \mathbf{v}_{\star}, \quad \rho=\int f \mathrm{~d} \mathbf{v} .
$$

Another reason for dealing with Maxwellian molecules is that we need "strong" entropy dissipation estimate to fulfil condition (31) (see below) and no such estimate is available for other cross-sections.

Theorem 3. Let $f \in L_{N}^{1}\left(\mathbb{R}^{3}\right)$ be a nonnegative function and $\mathbb{P}$ be a polynomial space of order $N$ satisfying (I),(II),(III), then the relaxation model

$$
R(f)=\rho(G-f)
$$

where $G$ is solution to the minimization problem:

$$
\min \left\{H(g) / \boldsymbol{\mu}(g) \leq^{*} \boldsymbol{\mu}\left(\rho^{-1} Q^{+}(f, f)\right)\right\}
$$


fulfils the following properties:

$$
\begin{gathered}
\langle R(f) m(\mathbf{v})\rangle=\langle Q(f, f) m(\mathbf{v})\rangle, \quad \forall m \in \mathbb{P} \text { such that } d^{0} m \leq N-1, \\
\left\langle R(f)|\mathbf{v}|^{N}\right\rangle \leq\left\langle Q(f, f)|\mathbf{v}|^{N}\right\rangle .
\end{gathered}
$$

In particular the model is mass, momentum and energy preserving as long as $\mathbb{E}_{0} \subset \mathbb{P}$. Besides one has:

$$
G=f \Rightarrow f=\text { Maxwellian distribution }
$$

and finally the model is entropy dissipating

$$
\langle R(f) \ln f\rangle \leq 0 \quad \text { and }\langle R(f) \ln f\rangle=0 \Rightarrow f=G=M .
$$

Proof. The first part of the theorem is essentially an application of Theorem 1. We just have to check that all assumptions are fulfilled: $\rho^{-1} Q^{+}(f, f) \geq 0$ a.e. is obvious since $f$ is nonnegative. Its moments are bounded thanks to the Povzner inequality [13]:

$$
\int_{\mathbb{R}^{3}}\left(1+|\mathbf{v}|^{2}\right)^{\frac{N}{2}} Q(f, f) \mathrm{d} \mathbf{v} \leq C|| f\left\|_{L_{N}^{1}}|| f\right\|_{L^{1}}
$$

with $C$ independent of $f$. So Theorem 1 directly applies and gives $(28,29)$ and $G$ is nothing but the entropic approximation of $\rho^{-1} Q^{+}(f, f)$ on $\exp (\mathbb{P})$. In particular

$$
H(G) \leq H\left(\rho^{-1} Q^{+}(f, f)\right) .
$$

Relations (30) and (31) are then a consequence of the absolutely nontrivial inequality:

$$
H\left(\rho^{-1} Q^{+}(f, f)\right) \leq H(f)
$$

which holds only for Maxwellian molecules. This last inequality is due in its most general form to Villani [15] (see therein for other references). Indeed as concerns the dissipation law (31) one just has to write

$$
\langle(G-f) \ln f\rangle \leq H(G)-H(f) \leq H\left(\rho^{-1} Q^{+}(f, f)\right)-H(f) \leq 0
$$

where we have used the convexity of $H$ and the inequality (32). Now remark that one can also write

$$
H\left(\rho^{-1} Q^{+}(G, G)\right) \leq H(G)
$$

so that in the case where $G=f$ one has $H\left(\rho^{-1} Q^{+}(f, f)\right) \leq H(G)$. But $G$ itself is the entropic approximation of $\rho^{-1} Q^{+}(f, f)$ so that

$$
G=\rho^{-1} Q^{+}(f, f)=\rho^{-1} Q^{+}(G, G)
$$

Multiplying this equality by $\ln G$ and integrating over $\mathbb{R}^{3}$ gives

$$
\int_{\mathbb{R}^{3}}\left(Q^{+}(G, G)-\rho G\right) \ln G \mathrm{~d} \mathbf{v}=\int_{\mathbb{R}^{3}} Q(G, G) \ln G \mathrm{~d} \mathbf{v}=0
$$

which holds only for Maxwellian distributions and proves (30). Finally by using (33)

$$
\langle(G-f) \ln f\rangle=0 \Rightarrow H(G)=H\left(\rho^{-1} Q^{+}(f, f)\right) \Rightarrow G=\rho^{-1} Q^{+}(f, f)
$$

and the same reasoning applies for characterizing stationary states. 
Remark that the main obstacle in extending the relaxation model (26) to general cross-sections is the inequality (32): in the general case no inequality of that type is available so that entropy decay is not ensured.

We finish this section by studying the asymptotic fluid limit of the non-dimensional equation:

$$
\partial_{t} f_{\epsilon}+\mathbf{v} \cdot \nabla f_{\epsilon}=\frac{\rho}{\epsilon}\left(G-f_{\epsilon}\right)
$$

where $G$ is defined above. In this section we shall assume that the space of moments $\mathbb{P}$ which defines $G$ uniquely through the minimization problem $(27)$ contains $\mathbb{E}_{0}=\operatorname{span}\left[1, v_{1}, v_{2}, v_{3},|\mathbf{v}|^{2}\right]-$ the space of collision invariants - together with the Sonine polynomials

$$
A_{i}(\mathbf{v})=\frac{1}{2}\left(|\mathbf{v}|^{2}-5\right) v_{i}, \quad B_{i j}(\mathbf{v})=v_{i} v_{j}-\frac{|\mathbf{v}|^{2}}{3} \delta_{i j}
$$

This means that $\mathbf{P}$ contains at least polynomials of degree 4 .

Suppose that $f_{\epsilon}$ can be expanded with respect to $\epsilon$ :

$$
f_{\epsilon}=f_{0}+\epsilon f_{1}+\epsilon^{2} f_{2}+\ldots
$$

Then we need to expand $G$ as well. Remark first that $G$ is a function of the moments of $Q^{+}$

$$
\langle G \mathbf{m}\rangle=\rho^{-1} \Pi_{h}\left(\left\langle Q^{+}(f, f) \mathbf{m}\right\rangle\right) \leq^{*} \rho^{-1}\left\langle Q^{+}(f, f) \mathbf{m}\right\rangle .
$$

(remember that the inequality can arise for the higher order moment) which according to the above expansion can be written:

$$
\langle G \mathbf{m}\rangle \leq^{*} \sum_{n=0}^{+\infty} \epsilon^{n} \boldsymbol{\rho}_{n}^{+} \text {where } \boldsymbol{\rho}_{n}^{+}=\rho^{-1} \sum_{i=0}^{n}\left\langle Q^{+}\left(f_{i}, f_{n-i}\right) \mathbf{m}\right\rangle .
$$

Let us now suppose that $G$ can be expanded as a function of its moments at $\boldsymbol{\rho}_{0}^{+}$:

$$
G\left(\boldsymbol{\rho}_{0}^{+}+\epsilon \boldsymbol{\rho}_{1}^{+}+\ldots\right)=G\left(\boldsymbol{\rho}_{0}^{+}\right)+\epsilon G^{\prime}\left(\boldsymbol{\rho}_{0}^{+}\right) \cdot \boldsymbol{\rho}_{1}^{+}+O\left(\epsilon^{2}\right) .
$$

Inserting this last expression and the expansion of $f$ in (34) and equating orders of $\epsilon$ gives:

- Order 1. $G\left(\boldsymbol{\rho}_{0}^{+}\right)=f_{0}$ and so according to (30) $f_{0}$ is a Maxwellian. We can choose the parameters of this Maxwellian $M$ so that its first moments match those of $f_{\epsilon}: \boldsymbol{\mu}_{0}\left(f_{\epsilon}\right)=\boldsymbol{\mu}_{0}(M)=\boldsymbol{\rho}_{0}^{+}$and so all other terms in the expansion are orthogonal to $\mathbb{E}_{0}$.

Remark now that $G$ has to be expanded around the moments of a Maxwellian distribution which justifies a posteriori the above expansion since $G$ is directionally differentiable at $\boldsymbol{\rho}_{M}$ :

$$
G^{\prime}\left(\boldsymbol{\rho}_{M}\right) \cdot \boldsymbol{\rho}=\lim _{\epsilon \rightarrow 0} \frac{1}{\epsilon}\left(G\left(\boldsymbol{\rho}_{M}+\epsilon \boldsymbol{\rho}\right)-G\left(\boldsymbol{\rho}_{M}\right)\right) \text { is well defined } \boldsymbol{\rho} \in \mathbb{R}^{\# P} .
$$

Besides $G(\boldsymbol{\rho})=\exp (\boldsymbol{\alpha} \cdot \mathbf{m}(\mathbf{v}))$ with $\boldsymbol{\alpha}=\tilde{h}_{\boldsymbol{\rho}}(\boldsymbol{\rho})\left(\tilde{h}\right.$ is itself directionally differentiable at $\left.\boldsymbol{\rho}_{M}\right)$ so that

$$
G^{\prime}\left(\boldsymbol{\rho}_{M}\right) \cdot \boldsymbol{\rho}=M \mathbf{m}(\mathbf{v})^{T} . \tilde{h}_{\boldsymbol{\rho} \boldsymbol{\rho}}\left(\boldsymbol{\rho}_{M}\right) \cdot \boldsymbol{\rho} .
$$

Remark here that the Hessian matrix $\tilde{h}_{\boldsymbol{\rho} \rho}\left(\boldsymbol{\rho}_{M}\right)$ is not single valued. It takes one form in the directions $\boldsymbol{\rho} \nsupseteq^{*} 0$ while it is degenerate in the case $0 \leq^{*} \boldsymbol{\rho}$ for which $\tilde{h}_{\boldsymbol{\rho} \rho}\left(\boldsymbol{\rho}_{M}\right) . \boldsymbol{\rho}=0$. In both cases we can however write

$$
\begin{gathered}
G\left(\boldsymbol{\rho}_{M}+\epsilon \boldsymbol{\rho}_{1}^{+}+\ldots\right)=M\left(1+\epsilon \mathbf{m}(\mathbf{v})^{T} \cdot \tilde{h}_{\boldsymbol{\rho} \boldsymbol{\rho}}\left(\boldsymbol{\rho}_{M}\right) \cdot\left(\sum_{n=1}^{+\infty} \epsilon^{n-1} \boldsymbol{\rho}_{n}^{+}\right)+O\left(\epsilon^{2}\right)\right) \\
=M\left(1+\epsilon \mathbf{m}(\mathbf{v})^{T} \cdot \tilde{h}_{\boldsymbol{\rho} \boldsymbol{\rho}}\left(\boldsymbol{\rho}_{M}\right) \cdot \boldsymbol{\rho}_{1}^{+}+O\left(\epsilon^{2}\right)\right) .
\end{gathered}
$$


On the other hand $G M^{-1}$ belongs to $L^{2}\left(\mathbb{R}^{3}, M\right)$ since either $G=M$ or $G$ is exponentially decaying with at least an order $0\left(\exp \left(-\alpha|\mathbf{v}|^{4}\right)\right)(\alpha>0)$. Therefore $G$ can be expanded on any (polynomial) Hilbert basis $\left(\phi_{n}\right)$ of $L^{2}\left(\mathbb{R}^{d}, M\right)$ :

$$
G(\mathbf{v})=M(\mathbf{v}) \sum_{n=0}^{+\infty}\left\langle G \phi_{n}\right\rangle \phi_{n}(\mathbf{v}) .
$$

Writing $\mathbf{m}$-the $\# P$-vector which components are vector basis of $\mathbb{P}$ - in terms of $\phi_{n}$ (this can always be done since $\phi_{n}$ is a polynomial basis) $G$ can be expanded as follows:

$$
G=M\langle G \mathbf{m}\rangle \cdot \mathbf{m}(\mathbf{v})+M \Sigma^{\perp}
$$

where

$$
\Sigma^{\perp}=\sum_{\phi_{n} \perp \mathbf{m}}\left\langle G \phi_{n}\right\rangle \phi_{n} \text { is orthogonal to } \mathbf{P} .
$$

Inserting (38) in this last expression proves that $\Sigma^{\perp}$ is of order 2 and

$$
G\left(\boldsymbol{\rho}_{M}+\epsilon \boldsymbol{\rho}_{1}^{+}+\ldots\right)=M\langle G \mathbf{m}\rangle \cdot \mathbf{m}(\mathbf{v})+O\left(\epsilon^{2}\right) .
$$

Expanding $\langle G \mathbf{m}\rangle$ as in (37) gives

$$
G\left(\boldsymbol{\rho}_{M}+\epsilon \boldsymbol{\rho}_{1}^{+}+\ldots\right)=M\left(1+\epsilon \tilde{\boldsymbol{\rho}}_{1}^{+} \cdot \mathbf{m}(\mathbf{v})+O\left(\epsilon^{2}\right)\right)
$$

with $\tilde{\boldsymbol{\rho}}_{1}^{+}=\boldsymbol{\rho}_{1}^{+}$in the non-degenerate case $\sum_{n \geq 1} \epsilon^{n-1} \boldsymbol{\rho}_{n}^{+} \Varangle^{*} 0$ and $\tilde{\boldsymbol{\rho}}_{1}^{+} \leq^{*} \boldsymbol{\rho}_{1}^{+}$in the degenerate case. Remark that in the degenerate case the (in)equality

$$
0 \leq^{*} \sum_{n \geq 1} \epsilon^{n-1} \boldsymbol{\rho}_{n}^{+} \Rightarrow\left(\boldsymbol{\rho}_{1}^{+}\right)_{i}=-\epsilon \sum_{n \geq 2} \epsilon^{n-2}\left(\boldsymbol{\rho}_{n}^{+}\right)_{i} \quad i=1, \ldots, \# P-1 .
$$

This means that all components of $\boldsymbol{\rho}+{ }_{1}$ (but the last one) must be themselves of order $\epsilon$.

- Order 0. Due to the above expansion the order 0 equation is:

$$
\partial_{t} f_{0}+\mathbf{v} \cdot \nabla f_{0}=\rho M\left(\tilde{\boldsymbol{\rho}}_{1}^{+} \cdot \mathbf{m}(\mathbf{v})-f_{1} M^{-1}\right) .
$$

Multiplying this equation with $1, \mathbf{v},|\mathbf{v}|^{2}$ gives the Euler system of equation since the right hand side of the equation is orthogonal to the collision invariants:

$$
\left\{\begin{array}{l}
\partial_{t} \rho+\nabla_{\mathbf{x}} \rho \mathbf{u}=0 \\
\partial_{t} \rho \mathbf{u}+\nabla_{\mathbf{x}}(\rho \mathbf{u} \otimes \mathbf{u}+p \mathcal{I})=0 \\
\partial_{t} \rho E+\nabla_{\mathbf{x}} \mathbf{u}(\rho E+p)=0
\end{array}\right.
$$

where

together with the closure relation:

$$
\rho=\int f \mathrm{~d} \mathbf{v}, \quad \rho \mathbf{u}=\int f \mathbf{v} \mathrm{d} \mathbf{v}, \quad \rho E=\frac{1}{2} \int f \mathbf{v}^{2} \mathrm{~d} \mathbf{v},
$$

$$
p=\frac{2}{3} \rho\left(E-1 / 2 \mathbf{u}^{2}\right)=\rho T .
$$

Differentiation of $M$ and using the Euler system of equations allows one to eliminate the time derivative in the left hand side of (40):

$$
\partial_{t} M+\mathbf{v} \cdot \nabla M=M\left(A(\mathbf{V}) \cdot \frac{\nabla_{x} T}{T^{1 / 2}}+B(\mathbf{V}): \nabla_{x} \mathbf{u}\right)
$$


where $A=\left(A_{i}\right)$ and $B=\left(B_{i j}\right)$ are defined in (35). Then (40) gives

$$
A(\mathbf{V}) \cdot \frac{\nabla_{x} T}{T^{1 / 2}}+B(\mathbf{V}): \nabla_{x} \mathbf{u}=\rho\left(\tilde{\boldsymbol{\rho}}_{1}^{+} \cdot \mathbf{m}(\mathbf{v})-f_{1} M^{-1}\right)
$$

which means that $f_{1} M^{-1} \in \mathbb{P}$ and one can write:

$$
f_{1}=M\left\langle f_{1} \mathbf{m}\right\rangle \cdot \mathbf{m}(\mathbf{v}) .
$$

Let us now focus on the right hand side of (43):

$$
\tilde{\boldsymbol{\rho}}_{1}^{+}-\left\langle f_{1} \mathbf{m}\right\rangle \leq^{*} \rho^{-1}\left\langle\left(2 Q^{+}\left(f_{0}, f_{1}\right)-\rho f_{1}\right) \mathbf{m}\right\rangle=2 \rho^{-1}\left\langle Q\left(M, f_{1}\right) \mathbf{m}\right\rangle
$$

which can be expressed in terms the linearized Boltzmann collision operator

$$
2\left\langle Q\left(M, f_{1}\right) \mathbf{m}\right\rangle=\left\langle M \mathcal{L}\left(f_{1} M^{-1}\right) \mathbf{m}\right\rangle \text { with } \mathbb{L}(\phi)=2 M^{-1} Q(M, M \phi) .
$$

This operator is self-adjoint for the scalar product in $L^{2}\left(\mathbb{R}^{3}, M\right)$ and its eigenvectors (generalized Hermite polynomials [7]) constitute precisely a Hilbert basis of $L_{M}^{2}$

$$
\left\langle M \mathcal{L}\left(f_{1} M^{-1}\right) \mathbf{m}\right\rangle=\left\langle f_{1} \mathcal{L}(\mathbf{m})\right\rangle=\Lambda \cdot\left\langle f_{1} \mathbf{m}\right\rangle
$$

where $\Lambda$ is a diagonal matrix composed of eigenvalues. The nullspace of $\mathcal{L}$ is $\mathbb{E}_{0}$ (collision invariants) and all other eigenvalues are negative. In particular

$$
\mathcal{L}\left(A_{i}(\mathbb{V})\right)=-\frac{1}{3} A_{i}(\mathbb{V}), \quad \mathcal{L}\left(B_{i j}(\mathbb{V})\right)=-\frac{1}{2} B_{i j}(\mathbb{V}), \quad \mathbb{V}=\frac{\mathbf{v}-\mathbf{u}}{T^{1 / 2}}
$$

and for the moment of highest degree

$$
\left(\tilde{\boldsymbol{\rho}}_{1}^{+}\right)_{\# P}-\left\langle f_{1} m_{\# P}\right\rangle \leq \rho^{-1} \lambda\left\langle f_{1} m_{\# P}\right\rangle \text { with } \lambda<0
$$

with equality in the non-degenerate case.

Inserting all those results in (40) proves that $f_{1}$ can simply be written as

$$
f_{1}=M\left(3 A(\mathbb{V}) \cdot \frac{\nabla_{x} T}{\sqrt{T}}+2 B(\mathbb{V}): \nabla_{x} \mathbf{u}\right)
$$

and that the Chapman-Enskog expansion of the density function $f_{\epsilon}$ at order 1 is exactly the same as the one for the full Boltzmann equation:

$$
f_{C E}^{B G K}=f_{C E}^{B o l t z}=M\left(1+3 \epsilon A(\mathbb{V}) \cdot \frac{\nabla_{x} T}{\sqrt{T}}+2 \epsilon B(\mathbb{V}): \nabla_{x} \mathbf{u}\right) .
$$

Consequently the order 1 equation gives after multiplication with $1, \mathbf{v},|\mathbf{v}|^{2}$ and integration over the velocity space the same Navier-Stokes equation - i.e. with same coefficients - as that obtained at the first order for the Boltzmann equation!:

$$
\left\{\begin{array}{l}
\partial_{t} \rho+\nabla_{\mathbf{x}} \rho \mathbf{u}=0 \\
\rho\left(\partial_{t}+\mathbf{u} \cdot \nabla_{\mathbf{x}}\right) \mathbf{u}+\nabla_{x}(\rho T)=\epsilon \nabla_{x} \cdot[\mu \sigma(\mathbf{u})] \\
\frac{3}{2} \rho\left(\partial_{t}+\mathbf{u} \cdot \nabla_{\mathbf{x}}\right) T+\rho T \nabla_{x} \mathbf{u}=\frac{\epsilon}{2} \mu \sigma(\mathbf{u}): \sigma(\mathbf{u})+\epsilon \nabla_{x} \cdot\left[\kappa \nabla_{x} T \sigma\right]
\end{array}\right.
$$


where $\mu=2 T$ is the viscosity and $\kappa=\frac{15}{2} T$ is the thermal diffusivity and $\sigma(\mathbf{u})$ is the strain-rate tensor given by

$$
\sigma_{i j}(\mathbf{u})=\frac{\partial u_{i}}{\partial x_{j}}+\frac{\partial u_{j}}{\partial x_{i}}-\frac{2}{3} \nabla_{x} \cdot \mathbf{u} \delta_{i j}
$$

Remark that here $\operatorname{Pr}=2 / 3$.

\section{Conclusion}

We have justified/developed an approximation tool that is well adapted to kinetic theory. It is essentially based on an entropy minimization principle with relaxed constraint(s) for the moments of highest degree. On one hand the approximation $f \rightarrow G$ conserves the positivity and a fixed number of moments of $f$ and on the other hand it is compatible with the entropy dissipation law - i.e. the approximation procedure does not contradict this law. There are essentially two possible issues to this work: theoretical and numerical. The first one is concerned with Levermore [11] moments closure hierarchy in kinetic theory. As remarked in Section 4 solutions to those systems suffer drawbacks when the moments of the solution are closed to the domain of nonrealizability, that is when there exists no exponential functions having those moments. We postpone to a future work possible modifications of those systems so as to make them compatible with the realizability problem. It shall then be interesting to connect solutions to those systems to solutions to the full Boltzmann equation.

Besides one may think of a Chapman-Enskog expansion where the approximate density is not a polynomial expansion (up to the multiplication of a local Maxwellian distribution) but an entropic approximation.

Finally numerical issues are also a possible continuation of this work especially as concerns our generalized BGK models. It is first interesting to try to extend those models to the case of general cross-sections and then to develop numerical algorithm that should improve computational costs. Such a generalization can be done for example on the basis of the paper of Mieussens [12] in the framework of discrete velocity models.

Acknowledgements. The author would like to thank J.J Alibert and G. Bouchitté for helpful discussions about convex analysis.

\section{REFERENCES}

[1] P. Andries, P. Le Tallec, J.P. Perlat and B. Perthame, The Gaussian-BGK model of Boltzmann equation with small Prandtl number. Eur. J. Mech. B Fluids 19 (2000) 813-830.

[2] L. Arkeryd, On the Boltzmann equation. Arch. Rational Mech. Anal. 45 (1972) 1-34.

[3] F. Bouchut, C. Bourdarias and B. Perthame, An example of MUSCL method satisfying all the entropy inequalities. C.R. Acad Sc. Paris, Serie I 317 (1993) 619-624.

[4] F. Coquel and P. LeFloch, An entropy satisfying muscl scheme for systems of conservation laws. Numerische Math. 74 (1996) $1-34$.

[5] I. Csiszár, I-divergence geometry of probability distributions and minimization problems Sanov property. Ann. Probab. 3 (1975) $146-158$.

[6] R. DiPerna and P.-L. Lions, On the Cauchy problem for Boltzmann equations: Global existence and weak stability. Ann. Math. 130 (1989) 321-366.

[7] H. Grad, On the kinetic theory of rarefied gases. Comm. Pure Appl. Math. 2 (1949) 331-407.

[8] M. Junk, Domain of definition of Levermore's five moments system. J. Stat. Phys. 93 (1998) 1143-1167.

[9] M. Junk, Maximum entropy for reduced moment problems. M3AS 10 (2000) 1001-1025.

[10] C. Léonard, Some results about entropic projections, in Stochastic Analysis and Mathematical Analysis, Vol. 50, Progr. Probab., Birkhaüser, Boston, MA (2001) 59-73.

[11] C.D. Levermore, Moment closure hierarchies for kinetic theories. J. Stat. Phys. 83 (1996) 1021-1065.

[12] L. Mieussens, Discrete velocity model and implicit scheme for the BGK equation of rarefied gas dynamics. Math. Models Methods Appl. Sci. 10 (2000) 1121-1149.

[13] A.J. Povzner, The Boltzmann equation in the kinetic theory of gases. Amer. Math. Soc. Trans. 47 (1965) 193-214.

[14] F. Rogier and J. Schneider, A Direct Method for Solving the Boltzmann Equation. Proc. Colloque Euromech n0287 Discrete Models in Fluid Dynamics, Transport Theory Statist. Phys. 23 (1994) 1-3.

[15] C. Villani, Fisher information bounds for Boltzmann's collision operator. J. Math. Pures Appl. 77 (1998) 821-837. 